\title{
PM Diagram of the Transfer Function and Its Use in the Design of Controllers
}

\author{
Santiago Garrido and Luis Moreno \\ Carlos III University of Madrid, Spain
}

Received: January 15, 2015 / Accepted: February 13, 2015 / Published: April 25, 2015.

\begin{abstract}
This paper presents an enhanced Root Locus diagram that consists of the representation of the phase and the magnitude of the transfer function $G(s)$ of a system. The magnitude is represented in decibels and the phase is represented by colors. It also presents how to use it to calculate different kinds of controllers. An important characteristic of this diagram is that permits to read the phase and the gain margins directly and the imaginary axis cut represents the Bode diagram. It is also possible to put the grid with damping ratio $\zeta$ and frequency $\omega n$.
\end{abstract}

Keywords: Root Locus, PID controller, Lead network, Bode plot, Phase margin, Gain margin.

\section{Introduction}

There are many possible representations of the transfer function $\mathrm{G}(\mathrm{s})$ of a system such as the Root Locus, the Bode diagram, the Nyquist diagram and the Nichols diagram. In all of them, it is possible to see some characteristics of the system and to create different kinds of controllers to improve the behavior of the system. Nowadays, with tools such as SISOTOOL of Matlab, it is possible to see many of these diagrams at the same time and the time response of the system to tune the controller.

The graphical representation of functions is one of the most important mathematical tools because it allows us to understand the behavior of the functions. While it is easy to represent the graph of a real function in the plane, the graph of a single variable complex function is more problematic. The reason adduced about why we cannot represent the Transfer Function is because $G(s): \rightarrow$ and as - is equivalent to represent two real variables, it is

Corresponding author: Santiago Garrido, Associate Professor in Robotics and Control Engineering, Ph.D. in Mathematics. Research field: Robotics and Control Engineering. E-mail: sgarrido@ing.uc3m.es. necessary to have 4 axes to represent the Transfer Function $G(s)$. Our brain is trained to visualize objects in three spatial dimensions, while the graphs of complex functions live in a four-dimensional space. Hence most of us are unable to imagine such an object.

Complex functions have the reputation of being mysterious entities; seeing these strange objects may help to overcome the fear one might feel while dealing with them.

The Phase Magnitude (PM) diagram makes it possible to put a face to the functions, deepening our intuitive understanding of basic and advanced concepts in complex analysis. They reveal intrinsic structures behind the formulas, literally open our eyes to the wonderful realm of complex functions, and may serve students, teachers, scientists, and engineers as simple and efficient tools in their work.

In Complex Variables papers [2], [3], [5] and books [4], [6], [7], and in related webpages, the researchers have represented complex functions in many ways that include the inclusion of the phase in the coloring. The problem, in general is that these representations do not permit measurements. 


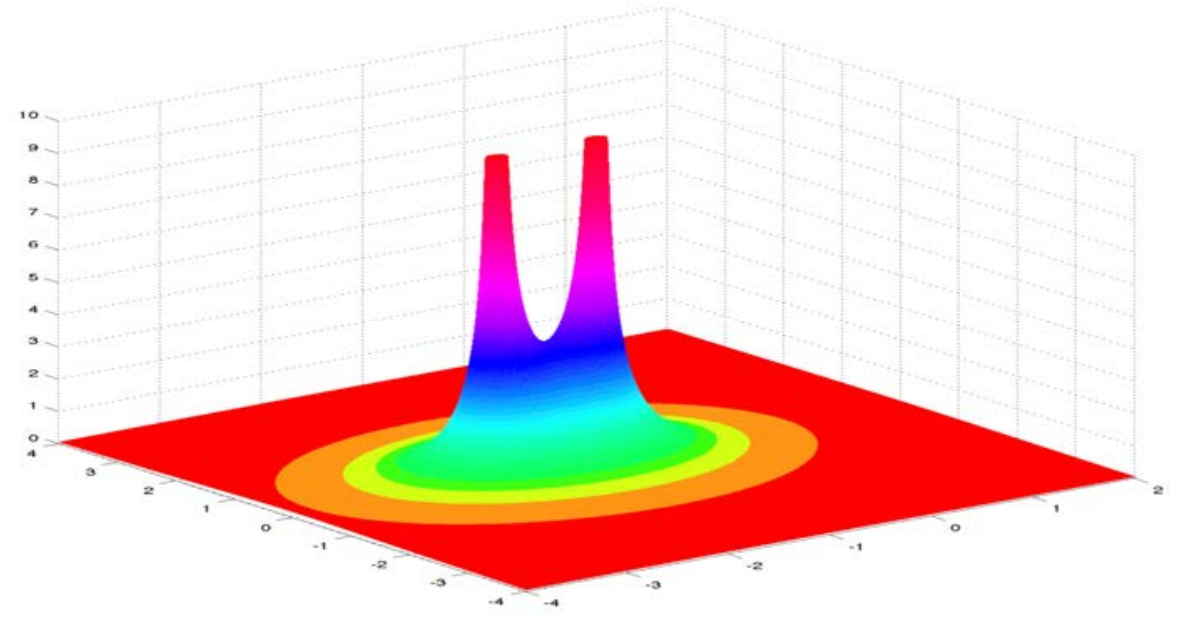

(a) $|G(s)|$

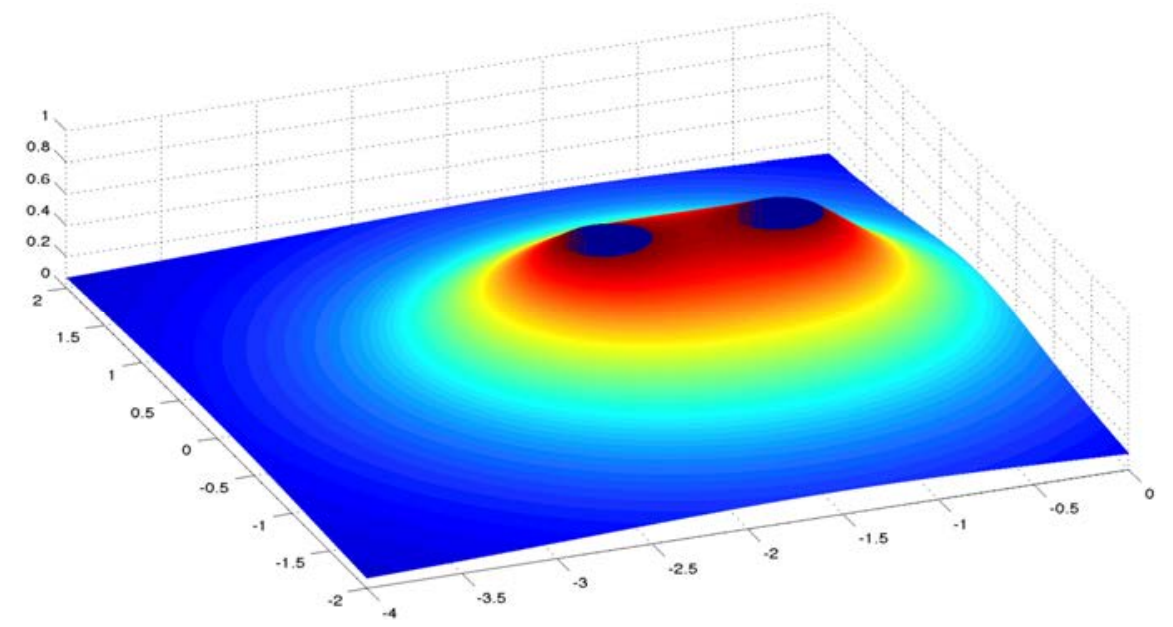

(b) $\arctan |\mathrm{G}(\mathrm{s})|$

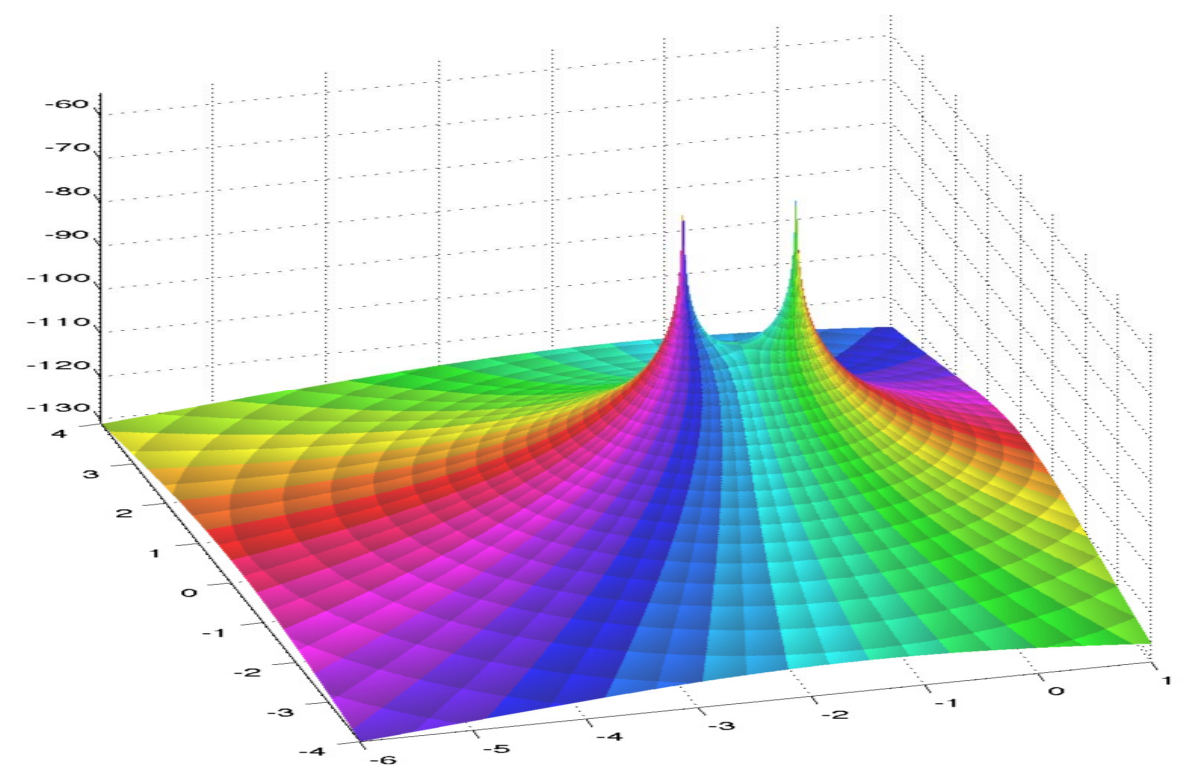

(c) $|\mathrm{G}(\mathrm{s})|$ and $\arg (\mathrm{G}(\mathrm{s}))$

Fig. 1 Different representations of a transfer function with two poles. 
Another possibility is to represent the magnitude and the phase of the function in each point with level curves. Cavicchi[8] tried this solution in 1996, but the problem was the bad resolution and the difficulty of reading his diagrams.

The solution adopted in this paper is to represent the fourth spatial dimension using the color-coded values of the phase on the domain of the function.

Another problem with the representation of transfer functions is that they are rational and they have poles. In these points the function goes to infinity and far from the poles and zeros, the function is quite plane, as shown in Fig. 1a). In these conditions, the level curves are too close near the poles and zeros, and there are not level curves in the almost plane zones. A possible solution to solve this problem is the technique adopted in the Complex Variable books [4], [6], [7]: to represent the arctan of the function G(s), but in this case the magnitude level curves are not equally spaced and the diagram can't be used numerically, as shown in Fig. 1b). The best solution for Control applications is the solution adopted by Bode in its diagram: to use the vertical scale in decibels $\mathrm{y}=20 \log _{10}(\mathrm{x})$, because in this way the zones near the poles have less level curves and the level curves are equally spaced: the space between two consecutive magnitude level curves is the same in decibels, as shown in Fig. 1c). In Fig. 1, these tree ways of representing the module of $G(s)$ as a surface are shown: $G(s)$ in the left part of the figure, $\arctan |\mathrm{G}(\mathrm{s})|$ in the middle, and $20 \log _{10}|G(s)|$ in the right part.

The solution adopted in this paper is inspired in [4], but represents the complex function by its module in decibels: $20 \log _{10}|G(s)|$ and its phase in rescaled 'hsv' coded colors.

Although it is possible to add level curves in magnitude and phase, it gives a more visual effect to shade in grey using the sawtooth function. As the magnitude is represented in decibels, the magnitude curves are equally spaced, with increments of $2 \mathrm{~dB}$, and the phase is also equally spaced, with increments of $10 \mathrm{o}$ It is possible to read in the representation almost directly the gain and the phase that has to add the controller and use the values directly in the solution of problems and exercises.

In Fig. 2 are shown the PM diagram of a single zero, $G(s)=s$, a double zero, $G(s)=s^{2}$, and a triple zero $G(s)=s^{3}$. As it can be seen the order of the colors is the same as that the original complex plane -. In the case of a double zero, the different colors appear twice and in the case of a triple zero, the different colors appear three times.

In Fig. 3 are shown the PM diagram of a single pole $G(s)=1 / s$, a double pole $G(s)=1 / s^{2}$, and a triple pole $G(s)=1 / s^{3}$. The different colors appear once, twice and three times but in the opposite orientation.

In all the PM diagrams presented in this work, the magnitude or modulus of the transfer function $G(s)$ is represented in decibels, i.e., it is represented as $20 \log _{10}|G(s)|$. In this way, the difference between two consecutive lines is the same in decibels. The other set of level curves, the colored ones, represent the phase $\arg (\mathrm{G}(\mathrm{s}))$. In this case, the scale is linear with red representing $0^{\circ}$ and cyan representing $180^{\circ}$. That means that the cyan line is the Root Locus and the red line is the Inverse Root Locus.

An important characteristic of this diagram is that it permits to read the phase margin directly because it is the phase distance from the actual closed poles position following the same magnitude line until the intersection with the imaginary axis and the Gain Margin that it is the magnitude distance following the cyan line that represents $180^{\circ}$ until its intersection with the imaginary axis.

The imaginary axes cut represents the Bode diagram. It is also possible to put the grid with damping ratio $\zeta$ and the frequency $\omega_{n}$. In the drawings, we have used linear scales for the real and imaginary axis, but if there are poles and zeros placed in different decades, it would be better to put log scales. 

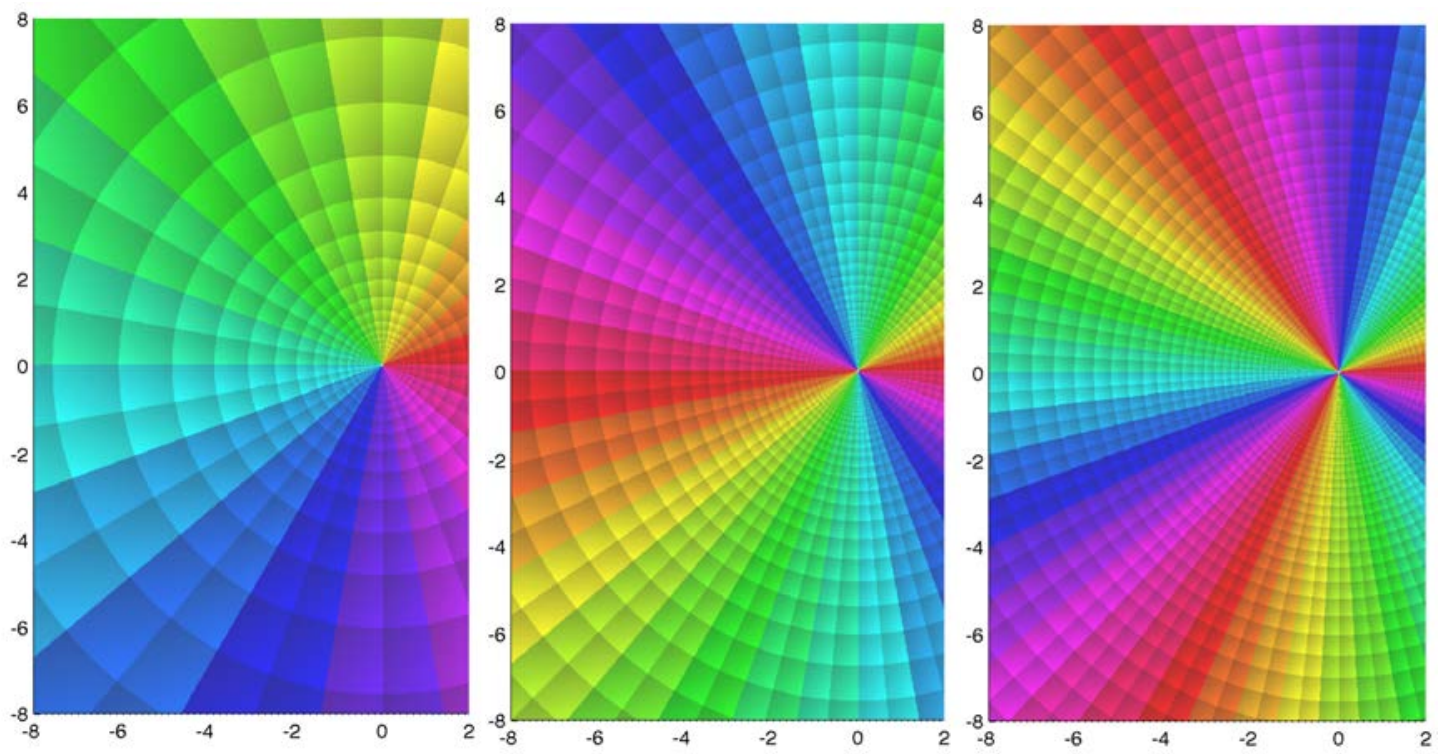

Fig. 2 PM diagram of a zero, a double zero and a triple zero.
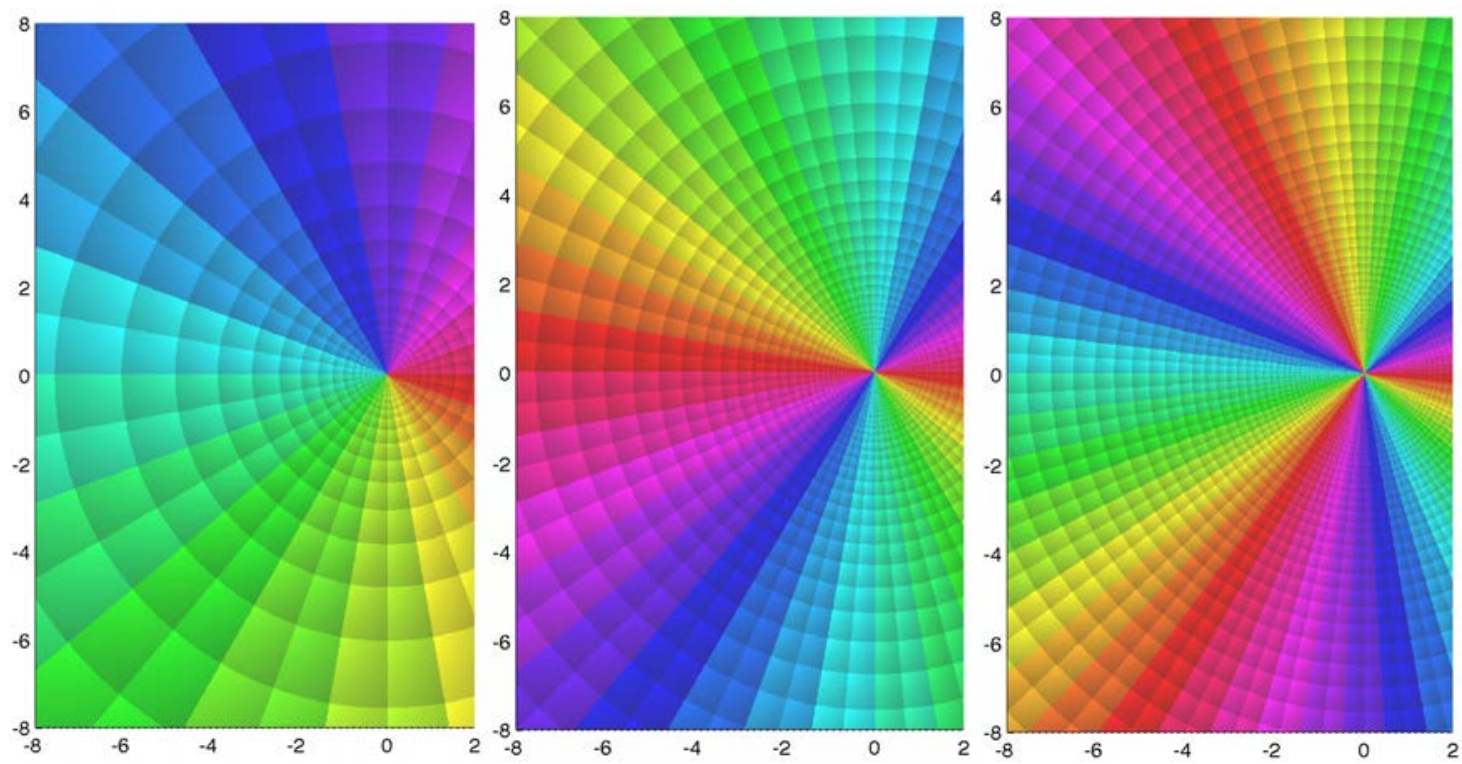

Fig. 3 PM diagram of a pole, a double pole and a triple pole.

In order to use these diagrams to calculate controllers $\mathrm{C}(\mathrm{s})$ for a system $\mathrm{G}(\mathrm{s})$, it is possible to read the phase, calculate the controller $\mathrm{C}_{1}(\mathrm{~s})$ with gain 1 that adds that phase, represent the PM diagram of $\mathrm{C}_{1}(\mathrm{~s}) \mathrm{G}(\mathrm{s})$, and then multiply by the gain needed to have $\mathrm{C}(\mathrm{s})=\mathrm{KcC}_{1}(\mathrm{~s})$.

The use of the SISOTOOL has a strange characteristic. You can add zeros and poles and change its positions, and change the gain until by trial and error you have the closed loop poles in the desired position. However, in the educational books about Control the process is different, you know the desired position of the closed loop poles and you have to calculate the controller in order to have the closed loop poles in the required positions. 1.1. Conformal Mapping

The expression conformal mapping means angle preserving and has been very important in history. In 1569, Mercator introduced the cylindrical map projection that is still in use for world maps. Another 

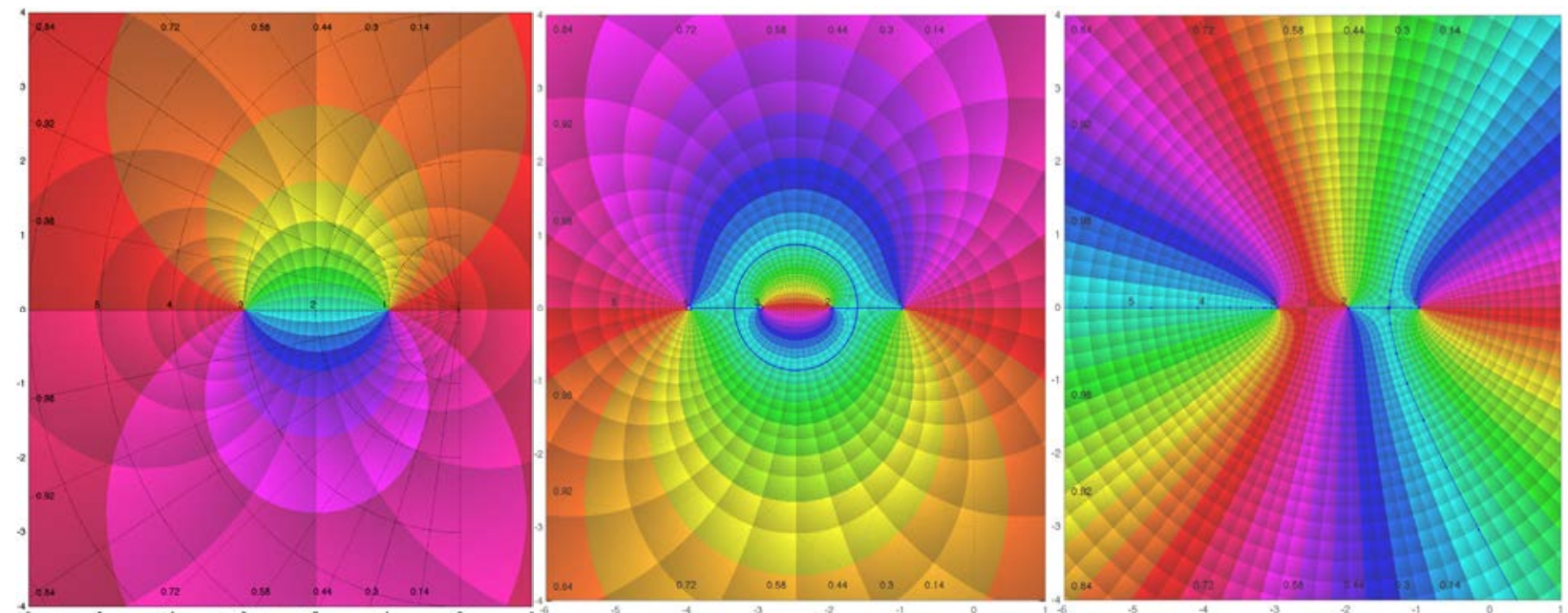

Fig. 4 PM diagram of different transfer functions.

(a) $G(s)=\frac{s+1}{s+3}$, (b) $G(s)=\frac{s^{2}+7 s+12}{s^{2}+3 s+2}$, (c) $G(s)=\frac{1}{(s+1)(s+2)(s+3)}$
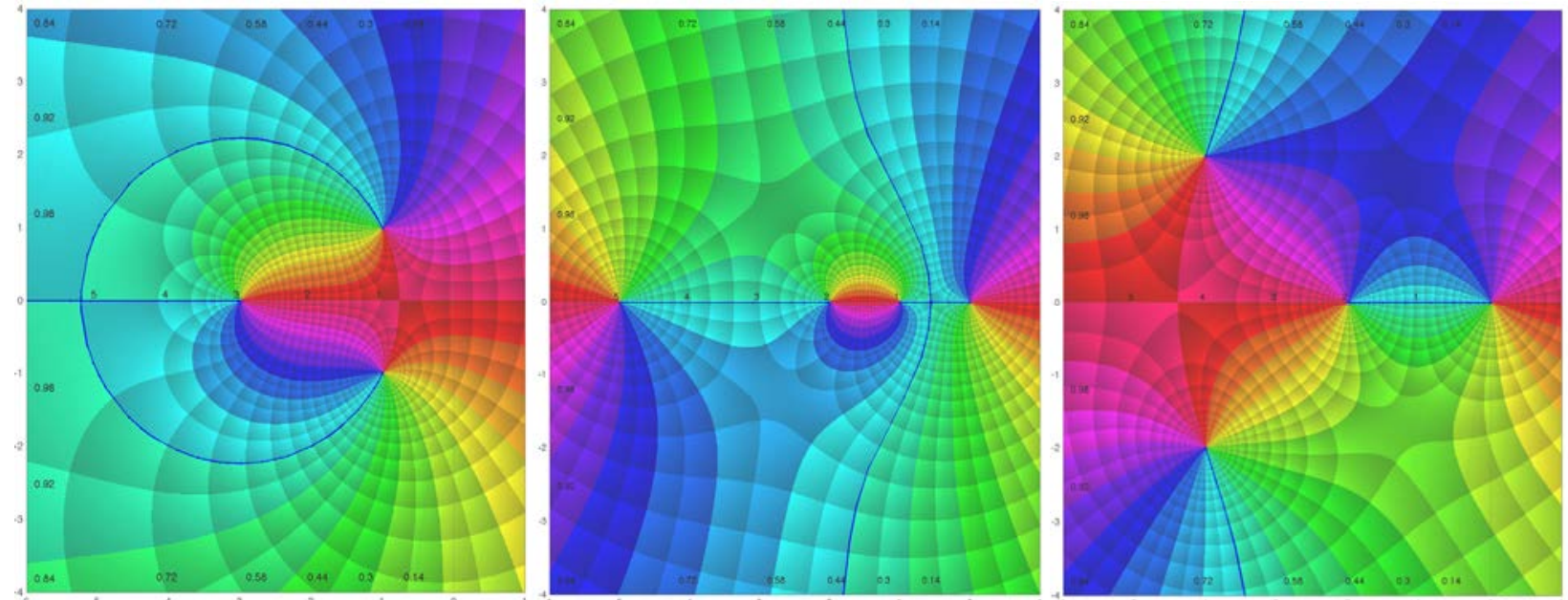

Fig. 5 PM diagram of different transfer functions.

(a) $G(s)=\frac{1}{(s+1)(s+2)}$, (b) $G(s)=\frac{s+2}{s\left(s^{2}+6 s+5\right)}$, (c) $G(s)=\frac{s+2}{s\left(s^{2}+8 s+20\right)}$

map projection is the stereographic projection introduced by the ancient greeks. In complex analysis, conformal mapping is a very important concept.

The transfer functions used in Control are rational functions that are analytic except in its poles. Consider an analytic function $\mathrm{f}$ in a domain $D$ and a point $z_{0}$ in D. If $f^{\prime}\left(z_{0}\right)=0$, then $f$ is conformal at $z_{0}$.

In effect, if $C_{1}$ and $C_{2}$ are two smooth curves passing trough $z_{0}$ with tangent vectors $v_{1}$ and $v_{2}$, and its respective inclination angles are $\beta_{1}$ and $\beta_{2}$ then the image curves $C_{1}^{\prime}$ and $C_{2}^{\prime}$ pass through $w_{0}=f\left(z_{0}\right)$, and have tangent

vectors $v_{1}$ and $v_{2}$ with inclination angles $\gamma_{1}$ and $\gamma_{2}$ related with $\beta_{1}$ and $\beta_{2}$ by the equations $\gamma_{1}=\alpha+\beta_{1}$ and $\gamma_{2}=\alpha+\beta_{2}$ where $\alpha=\arg \left(f^{\prime}\left(z_{0}\right)\right)$. Then, we conclude $\gamma_{2}$ $-\gamma_{1}=\beta_{2}-\beta_{1}$. That is the angle from $C_{1}$ to $C_{2}$ is equal to the angle from $C_{1}^{\prime}$ and $C_{2}^{\prime}$.

Therefore the transfer functions and its corresponding PM diagrams showed in the figures are conformal, and as the origin of the functions is the s-plane $\mathrm{C}$ shown in Fig. 2 and has right angles between the magnitude and phase level curves, then 
the angles of the image of the transfer function are also of $90^{\circ}$.

\section{Experimental Results}

\subsection{P design}

Consider the system $\mathrm{GM}=14.8 \mathrm{~dB}$ and suppose it is required a percent overshoot P.O. $\leq 10 \%$.

As P.O. $=\exp (-\pi / \tan (\theta)) \leq 0.10$, then $\theta \leq 53.76^{\circ}$, and the damping ratio is $\zeta \geq 0.5912$.

The real part of the desired closed loop poles is -1.5 and the imaginary part can be obtained as

$$
\begin{gathered}
\omega d=\sigma \tan \theta=1.5 \tan \left(53.76^{\circ}\right)=2.06 \\
\text { Using the angle condition } \\
\mathrm{K}=\frac{\Pi\left|s-p_{j}\right|}{\Pi\left|s-z_{i}\right|}=\sqrt{0.5^{2}+2.06^{2}} \sqrt{0.5^{2}+2.06^{2}}=4.48
\end{gathered}
$$

that means that the specification is satisfied for

$$
\text { Kc } \leq 4.48 \text {. }
$$

Using the PM diagram in Fig. 6, it is needed to click in the point of the Root Locus with damping ratio 0.59 and the corresponding value for $\mathrm{Kc}$ is 4.48 , i.e. the specification is satisfied for $\mathrm{Kc} \leq$ 4.48 .

\subsection{Ideal PD design}

Consider the system proposed before

$$
G(s)=\frac{1}{(s+1)(s+2)}
$$

This system has the closed loop poles placed in $\mathrm{s}_{1,2}$ $=-1.5 \pm 0.86 \mathrm{j}$ as shown in Fig. 7 (point on the right). Its magnitude is $|G(-1.5+0.86 j)|=1.0105$ and its phase is $\arg (\mathrm{G}(-1.5+0.86 \mathrm{j}))=180^{\circ}$

The desired specifications give us that the desired closed loop poles have to be placed in $\mathrm{s}_{1,2}=-3.14 \pm$ 3.14j, as shown in Fig. 7 (point on the left). The magnitude and phase of $\mathrm{G}(\mathrm{s})$ in these points are $|\mathrm{G}(-3.14 \pm 3.14 \mathrm{j})|=0.0788$ and $\arg (\mathrm{G}(-3.14 \pm 3.14 \mathrm{j}))$ $=125.77^{\circ}$.

If the desired controller is an ideal PD controller $\mathrm{C}(\mathrm{s})=\mathrm{Kc}(\mathrm{s}+\mathrm{b})$, then the phase can be calculated using Fig. 8 as

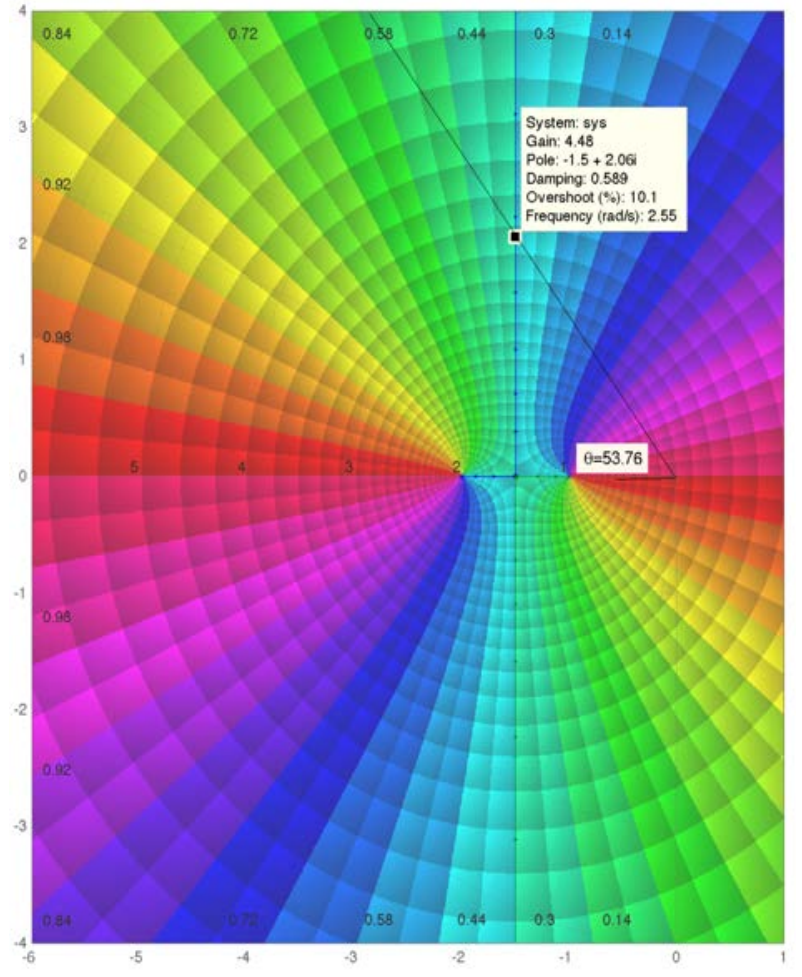

Fig. 6 PM diagram of $G(s)=\frac{1}{(s+1)(s+2)}$

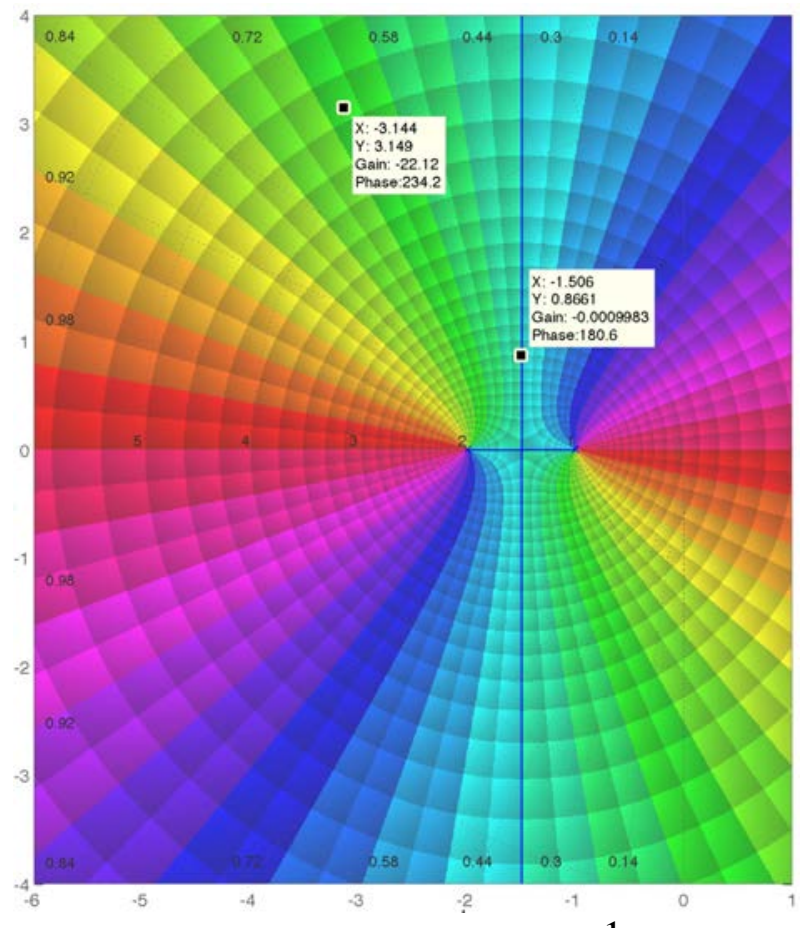

Fig. 7 PM diagram of $G(s)=\frac{1}{(s+1)(s+2)}$. The

original closed loop poles are $s_{1,2}=-1.5 \pm 0.86 \mathrm{j}$ (on the right), and the desired poles are $s_{1,2}=-3.14 \pm 3.14 \mathrm{j}$ (on the left). 


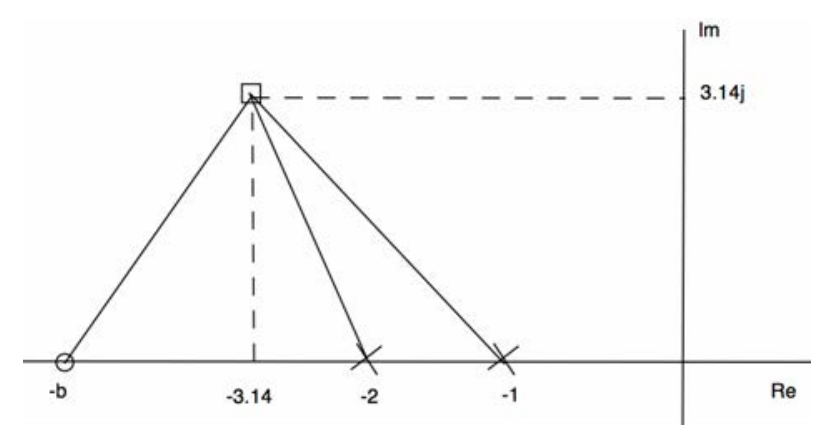

Fig. 8 Application of the phase condition for the ideal PD design.

$$
\begin{aligned}
& {\left[\arctan \frac{3.14}{b-3.14}\right]} \\
& -\left[180^{\circ}-\arctan \frac{3.14}{1.14}+180-\arctan \frac{3.14}{2.14}\right]=180^{\circ}
\end{aligned}
$$

i.e.

$$
\arctan \frac{3.14}{b-3.14}=54.2^{\circ}
$$

and that means that $\mathrm{b}=5.4$

In order to calculate the gain, the magnitude condition can be applied:

$$
K=\frac{d_{-1} d_{-2}}{d_{-5.4}}=\frac{\sqrt{3.14^{2}+1.14^{2}} \sqrt{3.14^{2}+2.14^{2}}}{\sqrt{3.14^{2}+(5.4-3.14)^{2}}}=3.28
$$

The desired controller is

$$
\begin{aligned}
& C(s)=K(s+b)=3.28(s+5.4) \\
& =K_{c} \frac{s+b}{b}=17.8 * \frac{s+5.4}{5.4}=17.8(1+0.19 s)
\end{aligned}
$$

The operations can be simplified using the PM diagram of the Figs. 7 and 9b:

The controller will be designed in two steps: The first step is the design of a controller of gain 1 that adds the desired phase (Fig. 7) and the second step is to calculate the necessary gain (Fig.9).

Between the cyan square and the green square in (Fig. 7) there are five and a half lines of phase (each one of $10^{\circ}$ ) and that means that the required phase of the controller has to be approximately $\varphi=54^{\circ}$. Obviously, the best way of measuring the angle is that it appears in the data tip, as shown in Fig. 7.

Applying $\arctan \frac{3.14}{b-3.14}=54.2^{\circ}$ the zero has to be in $b=-5.4$. The controller of gain 1 that adds the necessary phase is $C_{1}(s)=\frac{s+5.4}{5.4}=1+0.19 s$

Now, we represent the function

$C_{1}(s) G(s)=\frac{s+5.4}{5.4} \frac{1}{(s+1)(s+2)}$ as shown in Fig.

9. Now, we are going to design the second step: to calculate the necessary gain to pass from the point on right of Fig. 9b that are the actual closed loop poles to the point on the left that represent the desired poles.

The actual closed loop poles of this system are in $\mathrm{s}$ $=-1.59 \pm 0.68 \mathrm{j}$. In this figure, these closed loop poles are in the Root Locus (cyan line) and have magnitude $0 \mathrm{~dB}=1$. In order to find the gain, it is necessary to read the magnitude at the desired closed loop poles, $\left|\mathrm{C}_{1}(\mathrm{~s}) \mathrm{G}(\mathrm{s})\right| \mathrm{s}=-3.14+3.14 \mathrm{j}=-25 \mathrm{~dB}=0.056$, then

$\mathrm{Kc}=1 / 0.056=17.8$. The desired controller is $C(s)=K_{c} \frac{s+b}{b}=17.8 \frac{s+5.4}{5.4}=17.8(1+0.19 s)$

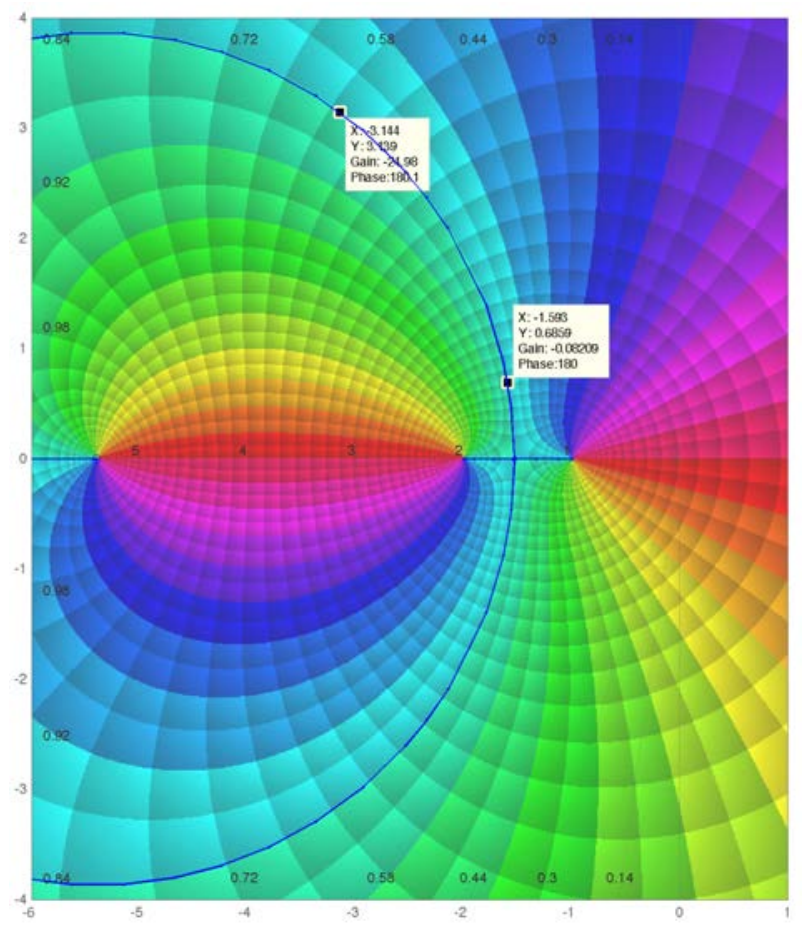

Fig. 9 Root Locus and PM diagram of the Transfer Function $G(s)=\frac{s+5.4}{5.4} \frac{1}{(s+1)(s+2)}$ of the ideal PD design. 


\subsection{Real PD design}

$G(s)=\frac{1}{(s+1)(s+2)}$

We will use the method proposed by Ogata[1] of calculate the bisector and to put the zero and the pole of the PD symmetrically to have the same angle between the pole and the bisector, and between the bisector and the zero, as shown in Fig. 10.

The PM diagram of G(s) is the same of the previous example, and it is shown in Fig. 7. The closed loop poles can be calculated as the solutions of the characteristic equation $1+\mathrm{G}(\mathrm{s})=0$. The poles are

$$
\mathrm{s}_{1,2}=-1.5 \pm 0.866 \mathrm{j} \text {. }
$$

We will use the same desired position of the closed loop poles as before: $\mathrm{s}_{1,2}=-3.14 \pm 3.14 \mathrm{j}$.

The $\arg (G(-3.14+3.14 j))=135^{\circ}$ and the angle deficiency that the real PD controller has to add is

Angle deficiency $=180^{\circ}-125.77^{\circ}=54.23^{\circ}$

Using the Fig.10 and placing the zero and the pole of the real PD controller symmetrically with respect to the bisector,

$$
\begin{gathered}
C(s)=K_{c} \frac{s+\frac{1}{T}}{s+\frac{1}{\alpha T}}=K_{c} \frac{s+4.9}{s+38.6} \\
K=\left|\frac{\Pi_{j}\left|\left(s-p_{j}\right)\right|}{\Pi_{i}\left|\left(s-z_{i}\right)\right|}\right|_{s=-3.14+3.14 j}=124.4 \\
=\left|\frac{(s+36.9)(s+1)(s+2)}{(s+4.6)}\right|_{s=-3.14+3.14 j}=15
\end{gathered}
$$

Using the magnitude condition

Finally, the desired real PD controller is

$$
C(s)=124.4 \frac{s+4.6}{s+36.9}=15.5 \frac{1+0.22 s}{1+0.027 s}
$$

The results of the PM and the Root Locus diagrams of the resulting system with the controller are shown in Fig.11.

An important remark is that it is possible to read the magnitude and phase of the controller directly from the PM diagram. Using this diagram, the example can

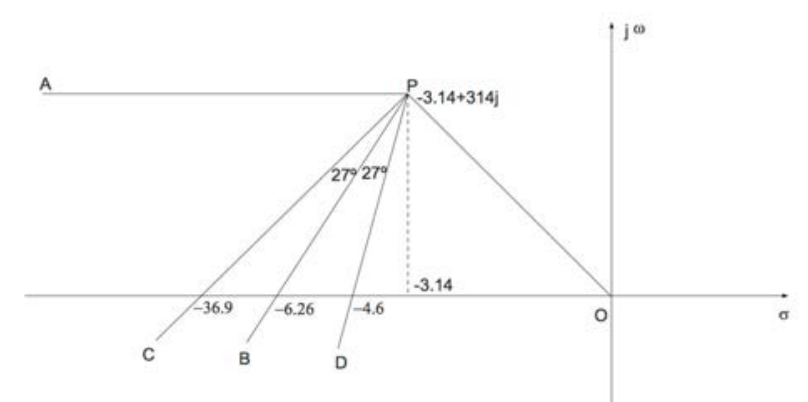

Fig. 10 Application of the angle condition for the real PD design.

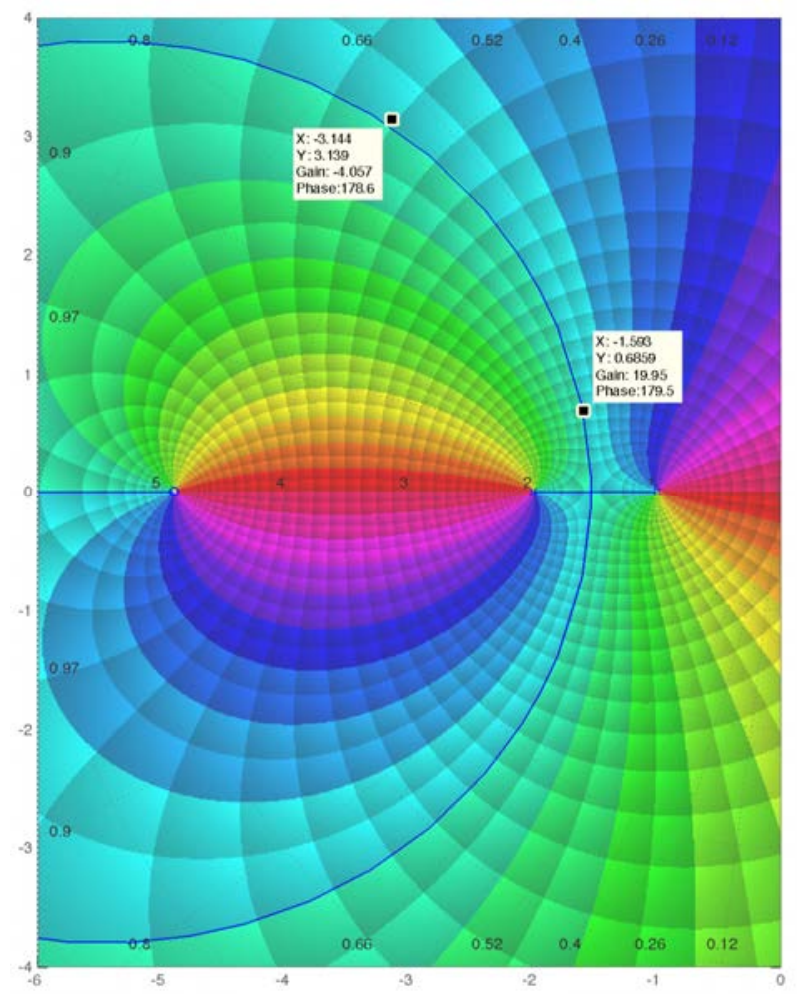

Fig. 11 Root Locus and PM diagram of the Tranfer Function $C(s) G(s)=\frac{38.6}{4.9} \frac{s+4.9}{s+38.6} \frac{10}{(s+1)(s+2)}$.

be solved in the following way. The actual closed loop poles of the system $G(s)=\frac{1}{(s+1)(s+2)}$ are $P_{a}=-1.5 \pm 0.866 j$. The desired dominant poles are $P_{d}=-3.14 \pm 3.14 j$. In Fig. 7 is possible to read that there are a bit more than 5 bands between that poles, each of $10^{\circ}$

$$
\text { Angle deficiency }=5.4 * 10^{\circ}=54^{\circ}
$$

Using Fig. 10, the controller is $C(s)=K_{c} C_{1}(s)=K_{c} \frac{38.6}{4.9} \frac{s+4.9}{s+38.6}$ 


\subsection{Design of a lead network}

Consider the system $G(s)=\frac{4 K}{s(s+2)}$

It is desired to find a controller with a static coefficient of velocity error $\mathrm{Kv}=20 \mathrm{~s}^{-1}$, and phase margin of $50^{\circ}$

The first step is to find the value of $\mathrm{K}$ in order to have the required $\mathrm{Kv}$

$$
K_{v}=\lim _{s \rightarrow 0} s G(s)=\lim _{s \rightarrow 0} s \frac{4 K}{s(s+2)}=2 K=20
$$

Therefore, $K=10$. Now, we represent the PM diagram of $G(s)=\frac{4 K}{s(s+2)}$ as shown in Fig.12. In this diagram, we find the point of the imaginary axis with magnitude line of $0 \mathrm{~dB}$, the phase margin is the number of phase bands between the actual position and that cyan band that represents 1800. In this case there are a bit less than two bands, approximately 180

$$
\text { The lead network } C(s)=\frac{1}{\alpha} \frac{s+z}{s+p}=\frac{1}{\alpha} \frac{s+\frac{1}{T}}{s+\frac{1}{\alpha T}}
$$

has to add a phase

$\phi=\gamma_{\text {desired }}-\gamma_{\text {actual }}+5^{\circ}=50^{\circ}-18^{\circ}+5^{\circ}=37^{\circ}$ and the parameter $\alpha$ of the network can be calculated as $\alpha=\frac{1-\sin (\phi)}{1+\sin (\phi)}=0.24$. As the lead network adds a magnitude of $10 \log _{10}(\alpha) d B$ in its middle point $\omega_{c}=\frac{1}{\sqrt{\alpha} T}$, we search the point with magnitude $K_{m}=10 \log _{10}(\alpha)=-6.2 d B$

This point correspond to frequency $\omega_{\mathrm{c}}=9 \mathrm{rad} / \mathrm{sec}$. Choosing this frequency as the new frequency of transition $\omega_{c}=\frac{1}{\sqrt{\alpha} T}$, it is possible to find the corner frequencies of the lead network $\frac{1}{T}=\sqrt{\alpha} \omega_{c}=4.41$ and $\frac{1}{\alpha T}=\frac{\omega_{c}}{\sqrt{\alpha}}=18.4$

In summary, the lead network is

$$
C(s)=\frac{18.4}{4.41} \frac{s+4.41}{s+18.4}=4.1723 \frac{s+4.41}{s+18.4}
$$

Where the multiplicative constant $\frac{1}{\alpha}$ is to have gain equal to 1 . The closed loop poles of the system with the lead network are placed in $-6.9 \pm 8 j$ as shown in Fig. 13.

\section{Phase Margin, Gain Margin and Bode diagram}

It is possible to put a 'grid' over the PM diagram in order to read the values of the damping ratio $\zeta$ and the natural frequency $\omega_{\mathrm{n}}$ as shown in Fig. 14 that represent the system $G(s)=\frac{11}{(s+1)(s+2)(s+3)}$.

In this case, the closed loop poles are placed in

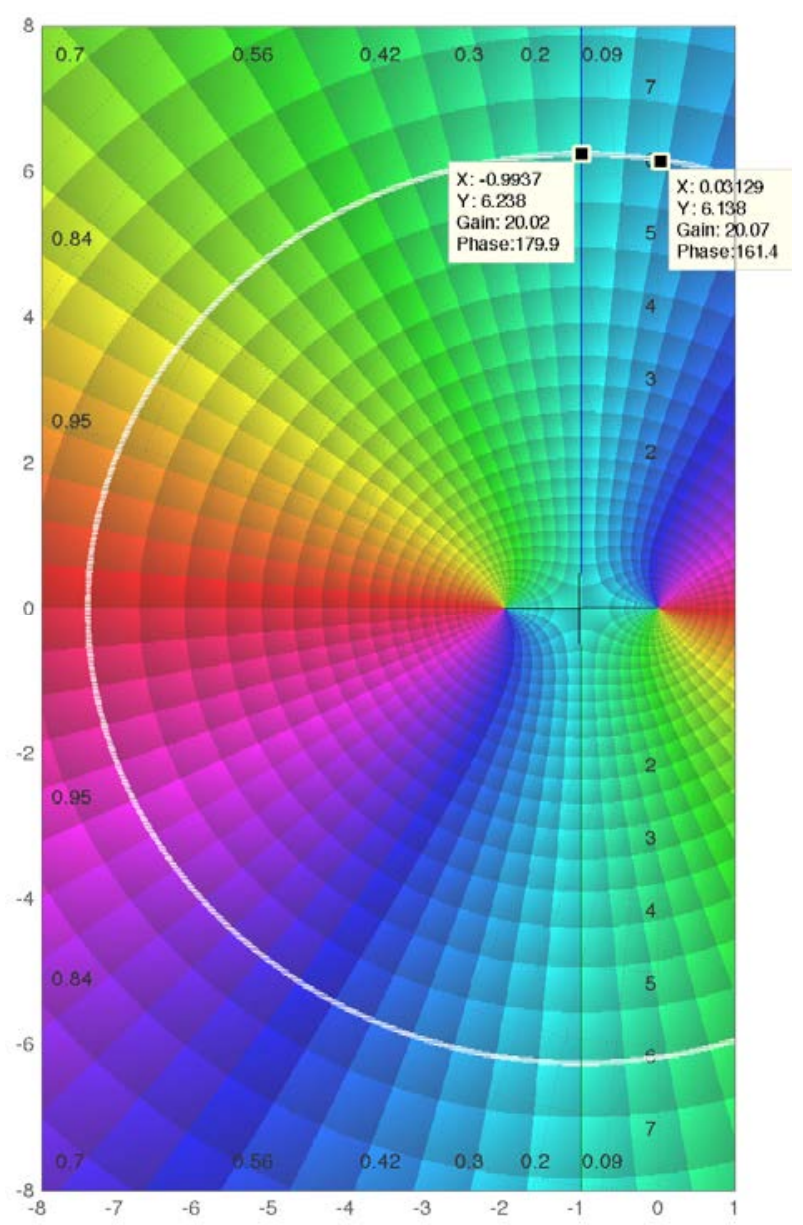

Fig. 12 PM diagram of

$C(s) G(s)=4.1723 \frac{s+4.41}{s+18.4} \frac{40}{s(s+2)}$. 


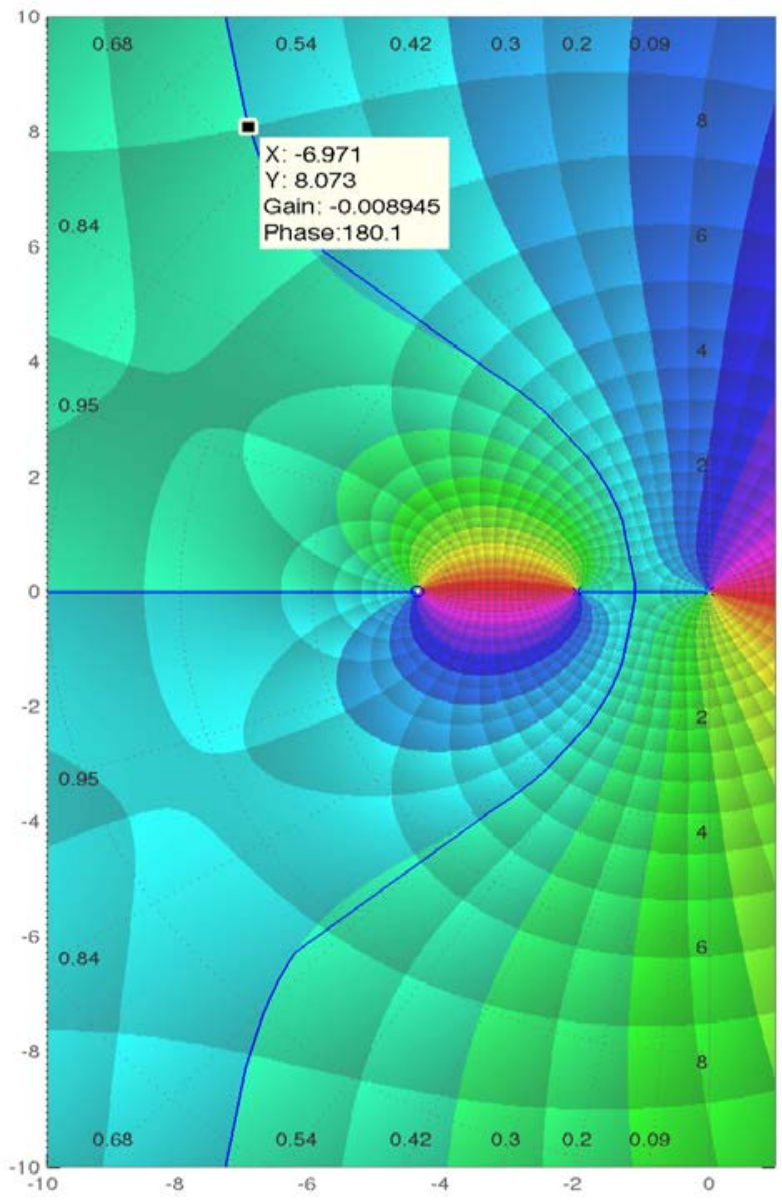

Fig. 13 Root Locus and PM diagram of

$$
C(s) G(s)=4.1723 \frac{s+4.41}{s+18.4} \frac{40}{s(s+2)} \text {. }
$$

$S_{1,2}=-0.8 \pm 1.8 j \quad, \quad$ because of this ||$G\left(s_{-}\{1,2\}\right) \mid=1=0 \mathrm{~dB}$. The cut of the imaginary axis is the Bode diagram. The gain margin is the value of the intersection of the Root Locus (cyan line) with the imaginary axis, in this case $\mathrm{GM}=14.8 \mathrm{~dB}$, and the Phase Margin is the number of color bands following the same zero gain line (white Line) until the intersection with the imaginary axis, multiplied by the value of each band, $10^{\circ}$. In this case there are 8 bands, approximately $\mathrm{PM}=80^{\circ}$.

Finally, in this figure the bandwidth (BW) is the distance over the imaginary axis from the origin to the point in which $|\mathrm{G}(\mathrm{s})|=-3 \mathrm{~dB}$. In this example, BW $=1.2 \mathrm{rad} / \mathrm{sec}$.

In the discrete case, the Root Locus and the PM diagram are represented in the same as in the

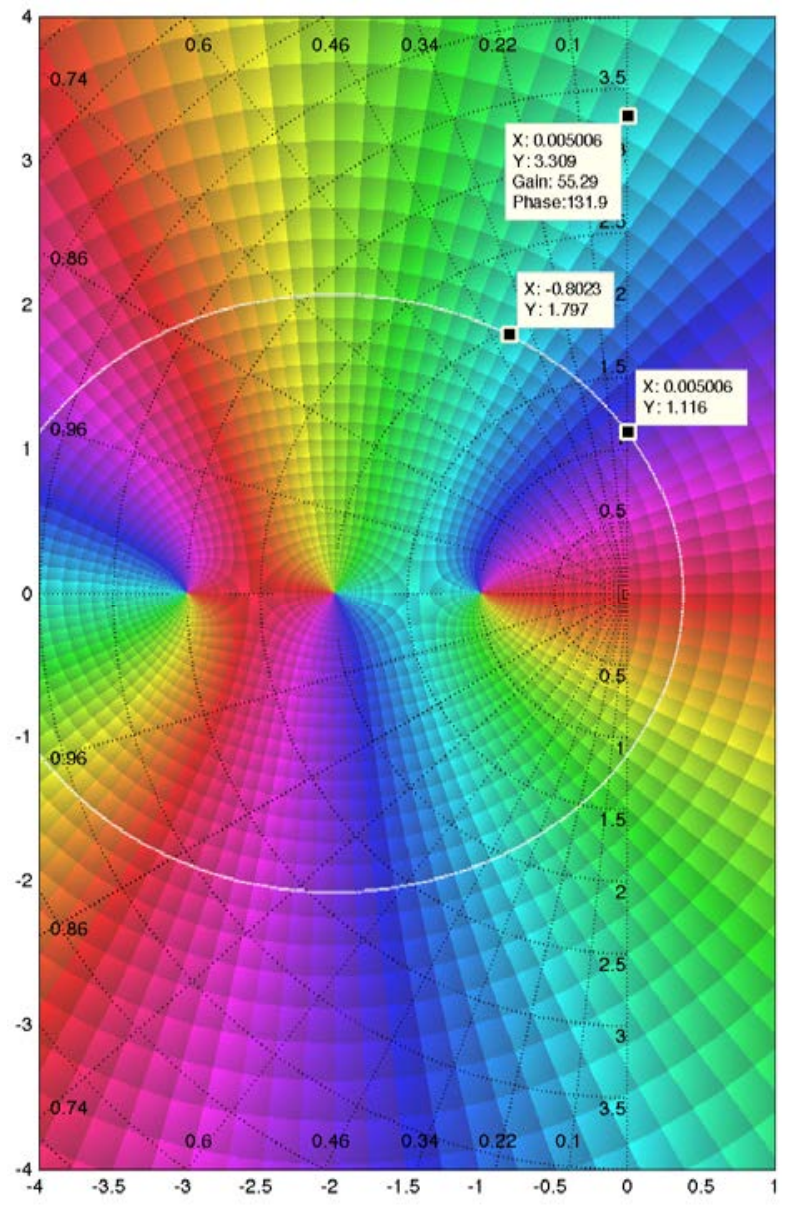

Fig. 14 PM diagram of the transfer function

$$
G(s)=\frac{11}{(s+1)(s+2)(s+3)} \text {. }
$$

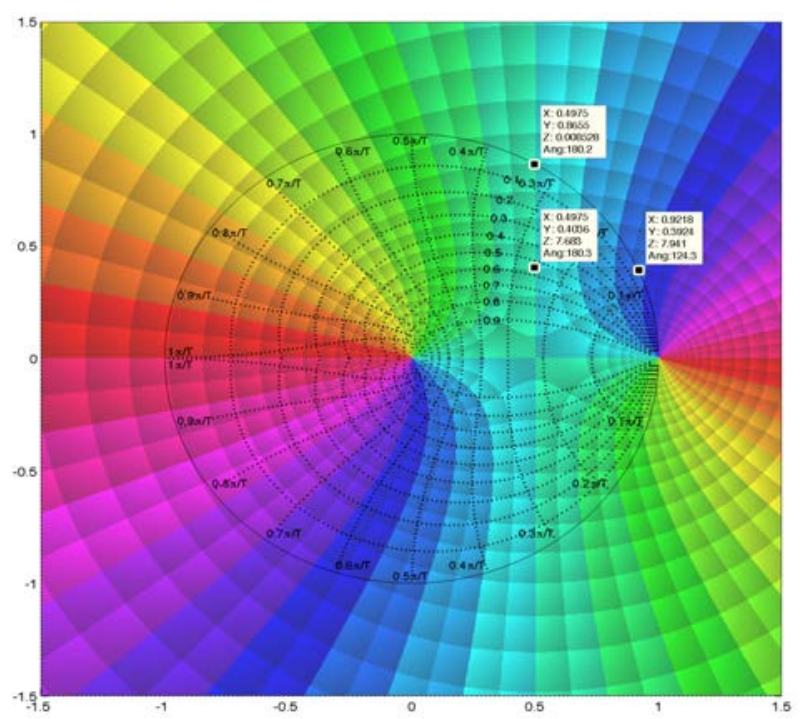

Fig. 15 PM diagram of the discrete transfer function

$$
G(z)=\frac{1}{z(z-1)} \text {. }
$$




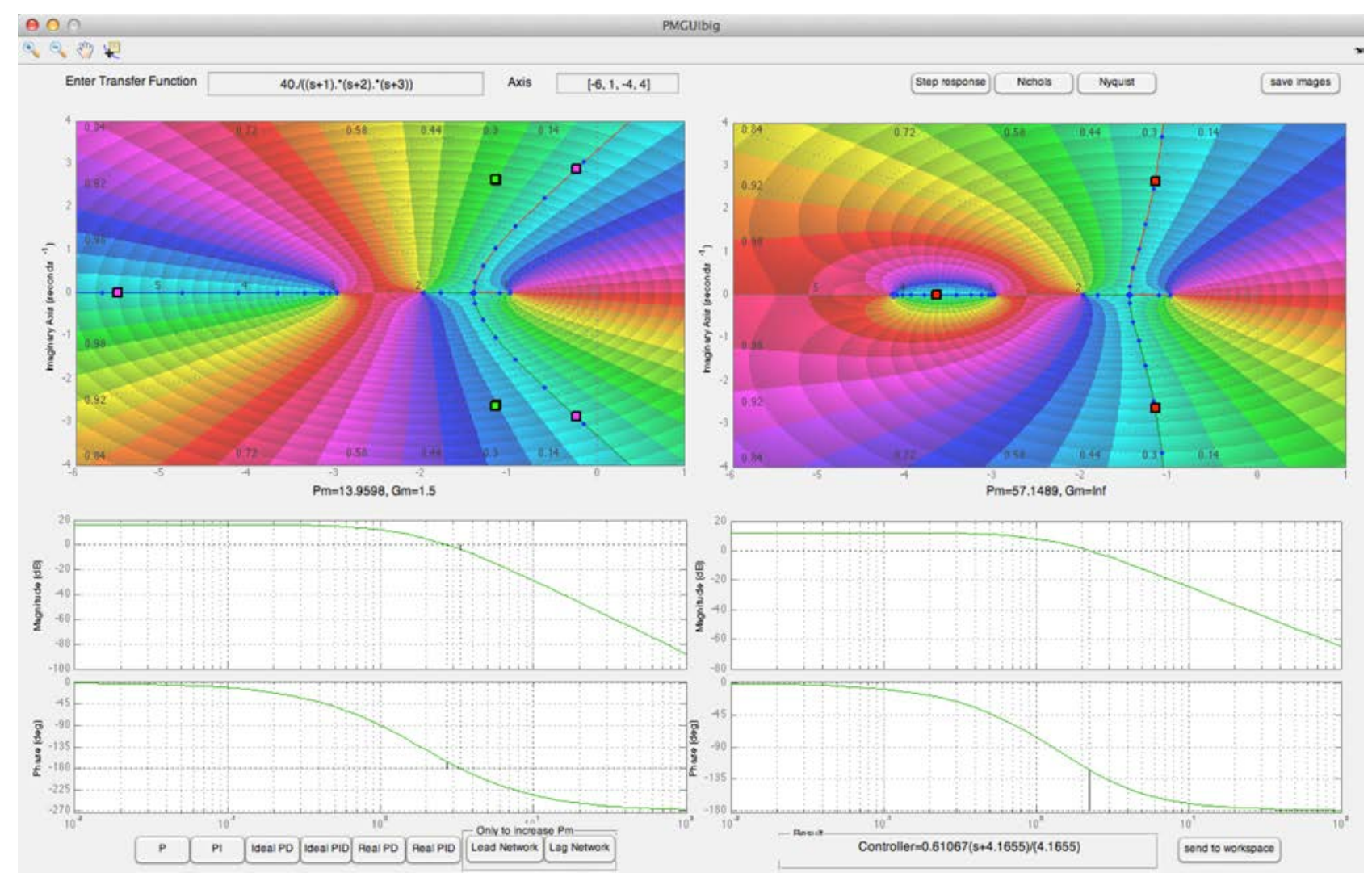

Fig. 16 PM diagram Graphic User Interface of the PM Diagram Matlab Toolbox

continuous case but the grid is different. Now, it is necessary to use the 'zgrid' Matlab command instead of the 'sgrid' command, as shown in Fig. 15 for the transfer function $G(z)=\frac{1}{z(z-1)}$. Suppose that the closed loop poles are in $0.5 \pm 0.4 \mathrm{j}$ as in the figure. That means that $|G(0.5 \pm 0.38 j)|=8 d B=2.5$, and the distance (in $|\mathrm{G}(\mathrm{z})|$ ) following the Root Locus (cyan line) to the intersection with the unit circumference is the Gain Margin, $\mathrm{GM}=8 \mathrm{~dB}=2.5$. The angle difference between the closed loop poles and the intersection of its magnitude level curve with the unit circumference is the Phase Margin, $P M=54^{\circ}$.

\section{Conclusions}

This paper present a new method of representation of transfer functions that it is an extension of the Root Locus and it is more visual and intuitive. It can be used to design controllers in an easier way and more intuitive than with the Root Locus diagram. It also give us an deeper knowledge of the transfer function and unifies the analysis in time and frequency because the cut of the PM diagram with the imaginary axis is the Bode diagram.

The Phase and Gain margins can be read directly in the PM diagram in a very visual and natural way, without using the Bode diagram. It also can be read the bandwidth.

Finally the PM diagram permits unify the time and frequency analysis. The authors have written a PM Diagram Toolbox for Matlab that include an Graphic User Interface shown in Fig. 16. This software is in the public domain and is available (at the time of this writing) from Matlab Central file exchange http://www.mathworks.es/matlabcentral/fileexchange/

\section{References}

[1] F. A. Farris (1997). "Visualizing complex-valued functions in the plane." AMC 10(12).

[2] E. Wegert, and G. Semmler (2010). "Phase plots of 
complex functions: a journey in illustration.” Notices AMS vol. 58. (768-780).

[3] E. Wegert (2012). Visual Complex Functions: An Introduction With Phase Portraits. Birkhuser.

[4] K. Poelke, and K. Polthier (2009). Lifted domain coloring. Computer Graphics Forum. Vol. 28. No. 3. Blackwell Publishing Ltd, 2009.
[5] T. Needham (1998). Visual Complex Analysis. Clarendon Press.

[6] J. Mathews, and R. Howell (2010). Complex Analysis for Mathematics and Engineering. Jones and Bartlett Learning.

[7] T. J.Cavicchi (1996). Phase-Root Locus and Relative Stability. IEEE control systems. 